\title{
Sensitive optical Microfiber-based Biosensors for DNA Detection
}

\author{
Rand Ismaeel, Timothy Lee, Tracy Melvin and Gilberto Brambilla \\ Optoelectronics Research Centre, University of Southampton, Southampton SO17 1BJ, United Kingdom
}

\begin{abstract}
We demonstrate an efficient method for DNA Sensing using simple devices, the microfiber coupler, and the Multi port Microcoil Resonator. These devices promise, high sensitivities, up to $200 \mathrm{~nm} / \mathrm{RIU}$, and low detection limit $\approx 10^{-6}$ RIU .
\end{abstract}

\section{Introduction}

In recent years, interest in biological sensors that utilize optical fibers have grown rapidly, as these fiberised sensors promise an efficient detection of the bio-molecules attached to the surface of the glass fiber. Unlike other types of sensors, these optical Biosensors are immune to electromagnetic interference, and have the ability to perform remote sensing in one simple integrated device.

Microfibers, on the other hand, with their attractive features of large evanescent field and low coupling losses, represent the ideal block element for future Biosensors. Devices that are fabricated with these Microfibers are compact, low loss and highly sensitive. The detection mechanism of these devices is based on the measurement of the refractive index (RI) change induced by the surface-molecule interaction, which is related to the sample concentration, Therefore, the detection sensitivity does not decrease with the volume of the measured sample. This last feature has made these sensors compatible for applications requiring small detection volumes [1]. Furthermore, both sensitivity and detection limit can be maximized by optimizing the device dimensions.

In General, DNA binding with silica introduces a refractive index change $\Delta \mathrm{n}$ in the range of $\approx 10^{-5}-10^{-2}$ for DNA concentrations between $0.5-2 \mu \mathrm{g} / \mathrm{mL}$ [1], which would then induce a wavelength shift in the transmission spectrum. Here, we present two types of microfiber devices for the purpose of DNA sensing: the microfiber coupler (MC) Fig.1 (a), and the 3D couler, which is Multiport Microcoil Resonator (MMCR) shown in Fig.1 (b) [2].

(a)

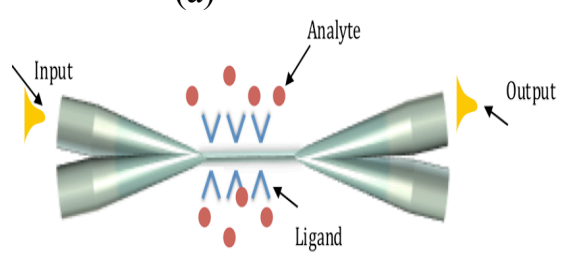

(b)

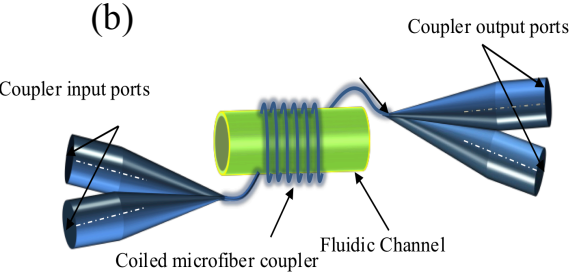

Fig. 1. (a) The microfiber coupler. (b) The multiport Microcoil Resonator with a fluidic channel.

\section{Theory}

The change in the indexis deduced by measuring the wavelength shift of both of the coupler and the MMCR transmission dip. Whereas changing the surrounding medium in the coupler will change the mode propagation condition inside the coupler waist, in the MMCR, changing the medium inside the channel will cause a shift in the resonance frequency to new values that corresponds to the concentration of the added DNA. The coupler coupling equations mentioned in [3] were solved and the results are shown in Fig.2 (a,b,c). The results showed the expected dependency of the coupler transmission dip on the DNA concentration, where high sensitivity could be obtained by carefully 
matching the coupler geometry with the DNA concentration being tested. Fig.2 (b) shows that this shift is dependent on the coupler radius, where higher sensitivity could be obtained for smaller couplers. However there is a limit to how small couplers can b,e as the smallers couplers have limited dynamic range and therefore can not be used to detect high DNA concentrations.
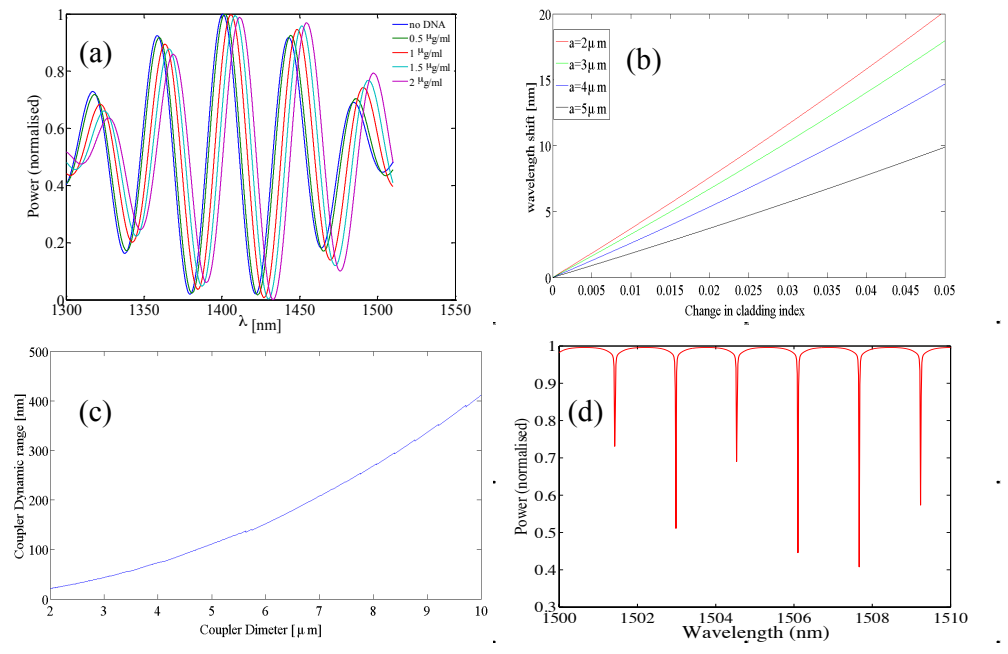

Fig. 2. (a) Different DNA concentration cause different shifts in the MC output. (b) Dependance of the shift on the MC diameter. (c) Dependance of the MC dynamic range on the coupler diameters.

(d) Spectrum of the MMCR shows high sensitvity.

As for the MMCR, the same equations for the MC are vaild, but since the coupler is coilled, the evanescent field coupling between the coil turns should be considered, and In order to simulate the transmission of the coilled MC, we assume $A_{n} L$ ) to be the amplitude of the electric field component in the $n^{\text {th }}$ turn of the coil at a distance $\mathrm{s}$ of the turn which has a total length L. $\beta$ represents the propagation constant of the mode $\beta=2 \pi n_{e f f} / \lambda$, the device transmission can be given by the following equations that describes the evolution of the electric field amplitudes $A_{j}(s)$ at a distance $\mathrm{s}$ along the turn which has a total length $\mathrm{L}[2]$.

$$
T=\frac{A_{n}(L) \exp (i \beta L)}{A_{1}(0)}
$$

A transmission spectrum of the device is shown in Fig. 2(d)The device shows extremely narrow resonances and a promising $\mathrm{Q}$ factor $>10^{8}$, thus forming the $\mathrm{MC}$ into a resonator, would not only increase the stability of the sensor, but it will makes it ideal for small concentration detection. Moreover, the sensor dynamic range could be maximised by enlarging the diameter of the fluidic channel.

\section{Experiment}

the $\mathrm{MC}$ was manufactured by pulling and streching two fibers together by using the modified flame brushing technique [4] , the MC dimensions were $5 \mu \mathrm{m}$ radius $20 \mathrm{~mm}$ Length.

The silanization process starts by inserting the coupler into a PTFE tube (the fluidic channel), which has a diameter of $1.5 \mathrm{~mm}$ and a volume of $10 \mu \mathrm{m}$. Substrates were cleaned by immersion in $1: 1$ concentrated $\mathrm{HCl}: \mathrm{MeOH}$ for 30 min, rinsed in deionized $\mathrm{H}_{2} \mathrm{O}$, therefore Silanol groups ( $\mathrm{SiOH}$ ) formed at the surface. The following step was to functionalize the coupler waist using a $1 \%$ solution of distilled trimethoxysilylpropyldiethylenetriamine in $1 \mathrm{mM}$ acetic acid in deionized (DI) $\mathrm{H}_{2} \mathrm{O}$ for $20 \mathrm{~min}$ at room temperature, rinsed three times with DI water and dried under $N_{2}$. The surface of the MC was modified with the heterobifunctional crosslinker, $\mathrm{N}$-(-maleimidobutryloxy) succinimide ester (GMBS), and prepared as $1 \mathrm{mM}$ solutions. Crosslinkers were dissolved in $100 \mu \mathrm{L}$ DMSO and diluted to $30 \mathrm{ml}$ in $80: 20 \mathrm{MeOH}: D M S O$. The silyl functionalised coupler was immersed in the GMBS crosslinker solution for 2 hours at room temperature, and then rinsed with the solvent used for dilution and dried under $N_{2}$. [5] The transmission of the coupler at this point represents a reference for the change that the DNA deposition would induce for the output 

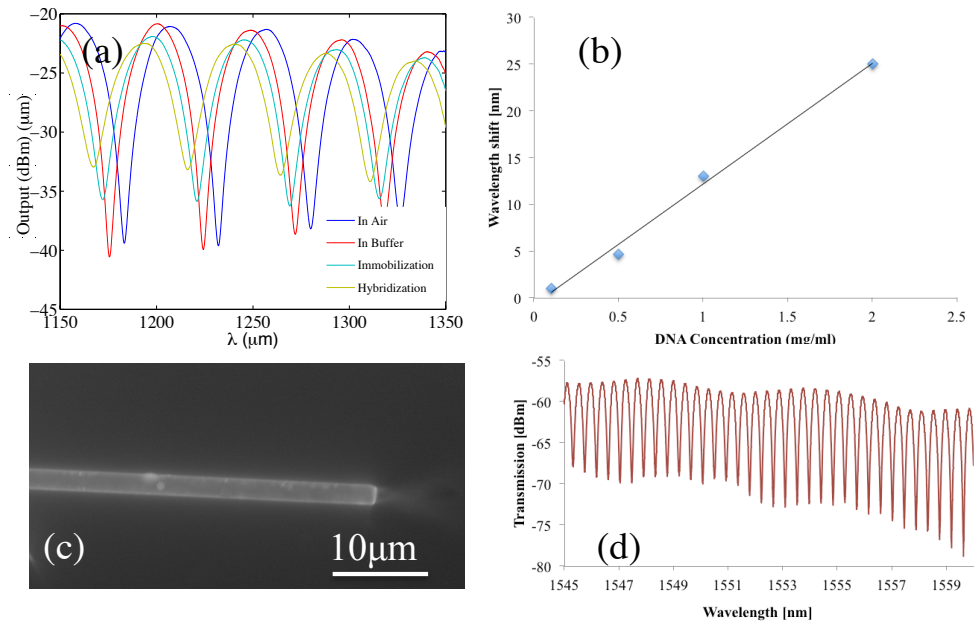

Fig. 3. (a) MC coupler: shifts in the spectra are associated to the coupler functionalization. (b) Dependence of the resonance shift on the DNA concentration (c) Microscope image confirming that the complimentary DNA strand is successfully attached to the coupler surface. (d) Spectrum of the MMCR: high Q-Factor device can be used to detect ultra-small quantites of bio molecules.

of the coupler. For the attachment of the DNA: 3-end labeled thiol-DNA oligonucleotide in deaerated 10mM HEPES, $5 \mathrm{mM}$ EDTA buffer (PH 6.6) was used. Concentrations of the DNA solution were of the order of $0.5-1 \mu \mathrm{g} / \mathrm{mL}$. Immobilization was complete by immersing the GMBS-functionalized coupler in a thiolated-DNA solution for 2 hours at room temperature, then rinsed and dried under $N_{2}$. For the attachment of the complimentary (hybridization) DNA: 3-e nd labeled thiol-DNA oligonucleotide deaerated 10mM HEPES, $5 \mathrm{mM}$ EDTA buffer. The MC spectrum was recorded along the different stages and its shown in Fig.3 (a).The complimentary DNA strand contains Florescence and a Florescence microscope was used to image the MC. The microscope image confirmed that the complimentary DNA strand was successfully attached to the coupler surface (Fig.3 (c)). Immobilization caused 4.7nm wavelength shift while hybridization caused an extra 5.2nm wavelength shift. Fig.3(b) shows the device response to different DNA concentrations, which in general shows a good match to the simulation results.

the MMCR was fabricated by coiling a MC around a PMMA tube which was then embeded in Teflon, PMMA was then carefully dissolved by acetone. This process produced a hollow Teflon cylinder that has the coiled microfiber in its inner surface, ready to be functionalized with biomaterial, Fig.3 (d) shows the high sensitivity spectrum of the hollow fluidic channel, this structure will be examined in the near future to detect ultra-small bio-molecules as wel as for DNA Immobilization and hybridization.

\section{References}

1. X. Fan, I. M. White, A. I. Shopova, H. Zhu, J. D. Suter, Sensitive optical biosensors for unlabeled targets: A review, Science direct analytica chimica acta 620, pp. 826 (2008).

2. R. Ismaeel, T. Lee, F. Al-Saab, Y. Jung, and G. Brambilla, "A self-coupling multi-port microcoil resonator," Opt. Express 20, 8568-8574 (2012).

3. R. Ismaeel, T. Lee, M. Gouveia, M. Ding, T. Melvin, and G. Brambilla, "Design and Optimization of a Microfiber Coupler for Biosensing," in Advanced Photonics 2013, K. Ewing and M. Ferreira, eds., OSA Technical Digest (online) (Optical Society of America, 2013), paper SM2D.3.

4. G. Brambilla, Optical fiber nanowires and microwires: a review, J. Opt., 043001 (2010).

5. L. A. Chrisey, G. U. Lee, .C. E. O'Ferrall, Covalent Attachment of Synthetic DNA to Self-Assembled Monolayer Films, Nucleic Acids Research 24, pp. 3031-3039 (2012). 